Preprint UGI-96-11

Submitted to Z. Phys. C

\title{
Probing Hadronic Polarizations with Dilepton Anisotropies *
}

\author{
E.L. Bratkovskaya, W. Cassing and U. Mosel \\ Institut für Theoretische Physik, Universität Giessen, D-35392 Giessen, Germany
}

\begin{abstract}
We investigate the production of $e^{+} e^{-}$pairs from $p+B e$ and nucleus-nucleus collisions from $2 \mathrm{GeV} / \mathrm{A}$ to $200 \mathrm{GeV} / \mathrm{A}$ within a covariant transport approach and focus on the dilepton angular anisotropies as a function of the dilepton invariant mass. Whereas the low mass regime yields information about the Dalitz decays of the $\Delta, \eta$ and $\omega$, above $\mathrm{M} \approx 0.5 \mathrm{GeV}$ the angular anisotropies provide additional information about the $\pi^{+} \pi^{-} \rightarrow \rho^{0} \rightarrow e^{+} e^{-}$channel in the medium. The anisotropy coefficient is found to change its sign for $\mathrm{M}>0.5 \mathrm{GeV}$ in case of nucleus-nucleus reactions when increasing the bombarding energy from $2 \mathrm{GeV} / \mathrm{A}$ to $200 \mathrm{GeV} / \mathrm{A}$ which sheds some light on the $\pi \pi \rightarrow \rho$ dynamics.
\end{abstract}

\footnotetext{
*Supported by BMBF and GSI Darmstadt
} 


\section{Introduction}

Nowadays, dileptons are used as electromagnetic signals from the hot and dense nuclear phase in heavy-ion collisions at BEVALAC/SIS [1, 2, 3] or SPS energies [4, 5, 6]. The information carried out by leptons may tell us not only about the interaction dynamics of colliding nuclei, but also on properties of hadrons in the nuclear environment or on a possible phase transition of hadrons into a quark-gluon plasma (cf. [7]). However, there are a lot of hadronic sources for dileptons because the electromagnetic field couples to all charges and magnetic moments. In particular, in hadron-hadron collisions, the $e^{+} e^{-}$pairs are created due to the electromagnetic decay of time-like virtual photons which can result from the bremsstrahlung process or from the decay of baryonic and mesonic resonances including the direct conversion of vector mesons into virtual photons in accordance with the vector dominance hypothesis. In the nuclear medium, the properties of these sources may be modified and it is thus very desirable to have experimental observables which allow to disentangle the various channels of dilepton production.

Apart from the differential $e^{+} e^{-}$spectra the investigation of lepton angular distributions is promising, too, because the virtual photon created in hadronic interactions is polarized. The coupling of a virtual photon to hadrons induces a dynamical spin alignment of both the resonances and the virtual photons due to the conservation laws and consequently the angular distribution of a lepton pair with respect to the polarized photon momentum is anisotropic.

The angular distributions of leptons for large invariant masses (above the $J / \Psi$ regime) were investigated at high energy, both experimentally [8] and theoretically (cf. the review of Strojnowski 9]), more than a decade ago. Here, the dominant source at large invariant masses is the quark annihilation (Drell-Yan) process. The former measurements allowed to observe a small deviation of the polar angular distribution from the prediction of the naive quark-parton model, the deviations observed, however, are in line with more detailed QCD computations. Thus the sensitivity of lepton angular characteristics already has been successfully used to differentiate between models.

More recently, it has been proposed [10, 11] to use the lepton pair angular distributions also for a distinction between different sources in the "small" invariant mass region $(M<$ $1 \mathrm{GeV}$ ), where a lot of dilepton sources contribute. It has been shown, furthermore, that due to the spin alignment of the virtual photon and the spins of the colliding or decaying hadrons, this lepton decay anisotropy turns out to be sensitive to the specific hadronic 
production channel. In our previous works [10, 11, 12] we have calculated the dilepton anisotropy for elementary nucleon-nucleon collisions and for $p+d$ reactions at BEVALAC energies. First results for proton-nucleus and nucleus-nucleus collisions at $1-2 \mathrm{GeV} / \mathrm{A}$ are reported in [13], where the calculated inclusive dilepton spectra were compared to the DLS data [1, 2, 3].

In this work we present a more systematic study on the dilepton angular anisotropy for $p+A$ and $A+A$ reactions from $2 \mathrm{GeV} / \mathrm{A}$ to $200 \mathrm{GeV} / \mathrm{A}$ within the covariant transport approach HSD [14]. At SPS energies the inclusive dilepton spectra can be compared to the

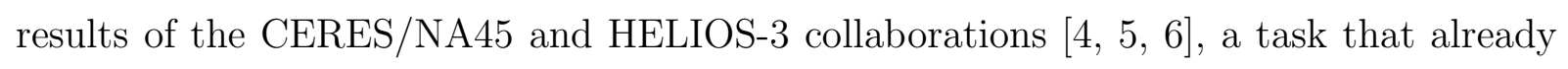
has been addressed with some success in [15, 16]. Here we proceed with predictions for the corresponding dilepton angular distributions and for the system $A u+A u$ at $10 \mathrm{GeV} / \mathrm{A}$ and $160 \mathrm{GeV} / \mathrm{A}$. Our work is organized as follows: in Section 2 we describe the evaluation of the lepton pair angular distribution and briefly repeat the main ingredients of the covariant transport approach. Section 3 contains the calculated results on dilepton spectra for $p+B e$ and various nucleus-nucleus collisions as well as the dilepton anisotropies as a function of the invariant dilepton mass. We summarize our study in Section 4 and present a brief discussion on the experimental requirements to measure the effects proposed.

\section{Theoretical ingredients}

\subsection{Definition of the anisotropy coefficient}

The general form of the angular distribution for the decay of a virtual photon into a lepton pair may be written [17] as

$$
\begin{gathered}
W(\theta, \varphi)=\frac{3}{8 \pi}\left[\rho_{11}\left(1+\cos ^{2} \theta\right)+\left(1-2 \rho_{11}\right) \sin ^{2} \theta+\rho_{1-1} \sin ^{2} \theta \cos ^{2} 2 \varphi\right. \\
\left.+\sqrt{2} \operatorname{Re} \rho_{10} \sin 2 \theta \cos \varphi\right]
\end{gathered}
$$

where the angles $\theta, \varphi$ of the electron momentum are measured with respect to a fixed $z$-axis in the virtual photon rest frame. The density matrix elements $\rho_{i j}$ depend on the choice of the reference frame as well as all the variables describing the virtual photon. An integration over the azimuthal angle gives

$$
W(\theta) \sim 1+B \cos ^{2} \theta,
$$


where the coefficient $B$ may vary between -1 and +1 . Adopting the normalization condition $\rho_{00}+2 \rho_{11}=1$, the coefficient $B$ can be represented as (cf. 18]):

$$
B=\frac{3 \rho_{11}-1}{1-\rho_{11}}
$$

Adopting the general form Eq. (1) the angular distribution of dileptons created in channel $i$ in a hadron-hadron $(h+h)$ reaction then can be written as [10, 11]

$$
\frac{d \sigma_{i}^{h h}}{d M d \cos \theta_{h h}}=A_{i}^{h h}\left(1+B_{i}^{h h} \cos ^{2} \theta_{h h}\right) .
$$

Here $\theta_{h h}$ is defined by $\theta_{h h}=\left(\overrightarrow{l_{-}^{*}}, \widehat{\vec{v}_{q}^{h h}}\right)$ with the electron momentum $\vec{l}_{-}^{*}$ measured in the dilepton center-of-mass system $\left(\vec{q}^{*} \equiv \vec{l}_{-}^{*}+\vec{l}_{+}^{*}=0\right)$, while $\vec{v}_{q}^{h h}=\vec{q}^{h h} / q_{0}^{h h}$ is the velocity of the dilepton c.m.s. relative to the $h+h$ c.m. system.

The coefficient $B_{i}^{h h}$ in Eq. (四) describes the anisotropy of the angular distribution ( $B_{i}^{h h}=0$ in case of isotropy), while $A_{i}^{h h}$ determines the magnitude of the respective cross section. The absolute value of $B_{i}^{h h}$ now depends on the choice of the coordinate system. For example, in the Gottfried-Jackson frame the $z$-axis coincides with the direction of the incident beam in the dilepton rest frame; this definition was adopted in the experiments before [8]. Following [10, 11] we will use for our analysis the "helicity" system, where the $z$-axis is chosen along the direction of the virtual photon momentum $\vec{q}$ in the center-ofmass system (c.m.s.) of the colliding hadrons.

The total differential cross section for $h+h$ collisions now can be represented as a sum of the differential cross sections for all channels $i$,

$$
\frac{d \sigma^{h h}}{d M d \cos \theta_{h h}}=\sum_{i=\text { channel }} \frac{d \sigma_{i}^{h h}}{d M d \cos \theta_{h h}}=A^{h h}(M)\left(1+B^{h h}(M) \cos ^{2} \theta_{h h}\right),
$$

which leads to the total anisotropy coefficient,

$$
B^{h h}(M)=\sum_{i=\text { channel }}<B_{i}^{h h}(M)>, \quad<B_{i}^{h h}(M)>=\frac{\frac{d \sigma_{i}^{h h}}{d M} \cdot \frac{B_{i}^{h h}}{1+\frac{1}{3} B_{i}^{h h}}}{\sum_{i} \frac{d \sigma_{i}^{h h}}{d M} \cdot \frac{1}{1+\frac{1}{3} B_{i}^{h h}}},
$$

where the special weighting factors originate from the necessary angle-integrations. Thus, the anisotropy coefficient $B^{h h}$ for $h+h$ reactions is the sum of the "weighted" anisotropy coefficients $\left(<B_{i}^{h h}>\right.$ ) for each channel $i$ obtained by means of the convolution of $B_{i}^{h h}$ with the corresponding invariant mass distribution (cf. [12]). 
The anisotropy coefficients for the bremsstrahlung, Dalitz decays of $\Delta$-resonance and $\eta$-meson in $p n$ and $p p$ interactions have been calculated on the basis of a one-bosonexchange model fitted to elastic $N N$ scattering in Ref. [11. These anisotropy coefficients are a function of the invariant mass $M$, the masses $m_{a}, m_{b}$ and the initial invariant energy $\sqrt{s}$ of the hadrons $a+b$ involved in the reaction. As shown in Ref. [10, the anisotropy coefficient for pion annihilation in the $\pi^{+} \pi^{-}$c.m.s. is given by $B_{\pi^{+} \pi^{-}}=-1$. For this particular situation, where there are only two particles in the initial and final states, $\theta_{h h}$ is the angle of the lepton momentum with respect to the pion momentum in the c.m.s. of the leptons (or pions), i.e. $\vec{q}^{*}=\vec{p}_{a}^{*}+\vec{p}_{b}^{*}=\vec{l}_{+}^{*}+\vec{l}_{-}^{*}=0$.

\subsection{Covariant transport approach for nucleus-nucleus collisions}

The dynamical evolution of proton-nucleus or nucleus-nucleus collisions is described by a coupled set of transport equations evolving phase-space distributions $f_{h}(x, p)$ for each hadron $h$ [14], i.e.

$$
\begin{aligned}
& \left\{\left(\Pi_{\mu}-\Pi_{\nu} \partial_{\mu}^{p} U_{h}^{\nu}-M_{h}^{*} \partial_{\mu}^{p} U_{h}^{S}\right) \partial_{x}^{\mu}+\left(\Pi_{\nu} \partial_{\mu}^{x} U_{h}^{\nu}+M_{h}^{*} \partial_{\mu}^{x} U_{h}^{S}\right) \partial_{p}^{\mu}\right\} f_{h}(x, p) \\
& \quad=\sum_{h_{2} h_{3} h_{4} \ldots} \int d 2 d 3 d 4 \ldots\left[G^{\dagger} G\right]_{12 \rightarrow 34 \ldots} \delta_{\Gamma}^{4}\left(\Pi+\Pi_{2}-\Pi_{3}-\Pi_{4} \ldots\right) \\
& \quad \times\left\{f_{h_{3}}\left(x, p_{3}\right) f_{h_{4}}\left(x, p_{4}\right) \bar{f}_{h}(x, p) \bar{f}_{h_{2}}\left(x, p_{2}\right)\right. \\
& \left.\quad-f_{h}(x, p) f_{h_{2}}\left(x, p_{2}\right) \bar{f}_{h_{3}}\left(x, p_{3}\right) \bar{f}_{h_{4}}\left(x, p_{4}\right)\right\} \ldots .
\end{aligned}
$$

In Eq. (7) $U_{h}^{S}(x, p)$ and $U_{h}^{\mu}(x, p)$ denote the real part of the scalar and vector hadron selfenergies, respectively, while $\left[G^{+} G\right]_{12 \rightarrow 34 \ldots} \delta^{4}\left(\Pi+\Pi_{2}-\Pi_{3}-\Pi_{4} \ldots\right)$ is the 'transition rate' for the process $1+2 \rightarrow 3+4+\ldots$ which is taken to be on-shell in the semiclassical limit adopted. The hadron quasi-particle properties in (7) are defined via the mass-shell constraint 19,

$$
\delta\left(\Pi_{\mu} \Pi^{\mu}-M_{h}^{* 2}\right)
$$

with effective masses and momenta given by

$$
\begin{aligned}
& M_{h}^{*}(x, p)=M_{h}+U_{h}^{S}(x, p) \\
& \Pi^{\mu}(x, p)=p^{\mu}-U_{h}^{\mu}(x, p)
\end{aligned}
$$

while the phase-space factors

$$
\bar{f}_{h}(x, p)=1 \pm f_{h}(x, p)
$$


are responsible for fermion Pauli-blocking or Bose enhancement, respectively, depending on the type of hadron in the final/initial channel. The dots in Eq. (7) stand for further contributions to the collision term with more than two hadrons in the final/initial channels. The transport approach (7) is fully specified by $U_{h}^{S}(x, p)$ and $U_{h}^{\mu}(x, p)(\mu=$ $0,1,2,3)$, which determine the mean-field propagation of the hadrons, and by the transition rates $G^{\dagger} G \delta^{4}(\ldots)$ in the collision term, that describe the scattering and hadron production/absorption rates.

In the HSDD approach the following baryon states are explicitly propagated: the nucleon, $\Delta, N^{*}(1440), N^{*}(1535), \Lambda, \Sigma$ and $\Xi$ as well as their antiparticles with proper isospin. The meson sector includes all pseudo-scalar mesons as well as the $1^{-}$vector-meson octett. For the baryon selfenergies we use the covariant parametrizations described in detail in [14, whereas all mesons $(m)$ in this work will be propagated without selfenergies, i.e. $U_{m}^{S}=U_{m}^{\mu}=0$. The inelastic transition rates are as described in Ref. [14, i.e. for invariant collision energies $\sqrt{s}<2.6 \mathrm{GeV}$ we employ explicite cross sections for baryon-baryon reactions as in the BUU approach of Ref. [20] whereas for $\sqrt{s} \geq 2.6 \mathrm{GeV}$ we employ the LUND string formation and fragmentation model [21] to describe the hadron production cross sections. As in [14] a string formation time $\tau=0.8 \mathrm{fm} / \mathrm{c}$ has been used to translate the elementary 'free' cross sections to hadron reaction rates. In case of meson - baryon reactions explicite cross sections - in line with the available experimental data - are taken into account for $\sqrt{s}<1.8 \mathrm{GeV}$ whereas the LUND model is used above $1.8 \mathrm{GeV}$ invariant collision energy. Meson - meson reactions, furthermore, are treated as binary collisions including Breit-Wigner resonance cross sections with resonance properties taken from [22].

The coupled set of transport equations (7) is solved within the testparticle method employing the parallel emsemble algorithm [23], where a hadron is represented by a single testparticle in each individual parallel ensemble. In this way the 4-momenta of all hadrons are known throughout the collision together with their inelastic and elastic transitions. We note that the HSD approach quite well describes the reaction dynamics for $p+A$ and $A+A$ collisions from SIS to SPS energies as demonstrated in [14].

The production of dilepton pairs $\left(e^{+} e^{-}\right)$includes the Dalitz decays $\Delta \rightarrow N e^{+} e^{-}, \eta \rightarrow$ $\gamma e^{+} e^{-}, \omega \rightarrow \pi^{0} e^{+} e^{-}$, the bremsstrahlung for charged $N N, \pi N$ and $\pi \pi$ collisions as well as the direct decays of the vector mesons $\rho, \omega$ and $\phi$. Furthermore, the secondary mesonic channels $\pi^{+} \pi^{-} \rightarrow \rho^{0} \rightarrow e^{+} e^{-}, K \bar{K} \rightarrow \phi \rightarrow e^{+} e^{-}$and $\pi \rho \rightarrow \phi \rightarrow e^{+} e^{-}$are taken into

\footnotetext{
${ }^{1}$ Hadron-String-Dynamics
} 
account, too. The decay ratios are taken from the particle data table 22] whereas the formfactors for the Dalitz decays are adopted from Landsberg [24]. Further details of the computations are given in Refs. [20, 15, 16] and don't have to be repeated here.

\subsection{Anisotropy coefficients for $p+A$ and $A+A$ reactions}

For heavy-ion reactions the situation becomes more complicated due to the nuclear dynamics and the explicit time evolution of the interacting system. For the calculation of the nuclear anisotropy coefficient we start from the point that the form of the angular distribution for all "elementary" hadron-hadron interactions $a+b$, that occur in the nucleus-nucleus reaction $A+B$, are known from the microscopic calculations [10, 11, 12].

In case of $\mathrm{A}+\mathrm{A}$ reactions we have to take into account that the "elementary" $a+b$ c.m.s. (in which the $B_{i}^{a b}$ are computed) are moving relative to the $A+B$ c.m.s. This implies that the direction of the virtual photon momentum $\vec{q}^{a b}$ relative to the hadronhadron c.m.s. $a+b$ is different for each elementary interaction $a+b$ and can't be restored from the experimental data. However, the direction of the virtual photon momentum $\vec{q}^{A B}$ in the nucleus-nucleus c.m.s. $A+B$ can be reconstructed for each lepton pair. Thus, it is necessary to perform an angular transformation from $\theta_{a b}$ to $\theta_{A B}$, where $\theta_{A B}$ is the angle between the lepton momentum $\overrightarrow{l^{*}}$ and the velocity of the dilepton c.m.s. relative to the c.m.s. of the colliding nuclei $A+B\left(\vec{v}_{q}=\vec{q}^{A B} / q_{0}^{A B}\right)$.

At high energies $(\sim 200 \mathrm{GeV} / \mathrm{A})$ the main contributions to the dilepton spectra come from the Dalitz decays of $\eta, \omega$-mesons, $\pi^{+} \pi^{-}$-annihilation and direct decay of vector mesons $\rho, \omega, \phi$. As shown in Ref. [15] the contributions from the $\Delta$-Dalitz decay and bremsstrahlung are negligible. Furthermore, the polarization of vector mesons created in direct nucleon-nucleon reactions is practically zero according to the measurements of Blobel et al. 25]. This is due to the fact that the exclusive cross section $N N \rightarrow N N \rho^{0}, \omega, \phi$ is very small compared to the inclusive cross section $N N \rightarrow N N \rho^{0}, \omega, \phi+X$ containing several pions in the final channel, too. Consequently, we do not consider the contribution to the anisotropy coefficient from the decays of vector mesons produced in primary $N N$-collisions. Thus, there are only three dominant channels at intermediate and high energies, i.e. the Dalitz decays of $\eta$ - and $\omega$ - mesons as well as $\pi^{+} \pi^{-}$annihilation that have to be taken into account in our analysis.

For the computation of the nuclear anisotropy coefficient we use another algorithm than in our previous paper [13], i.e. we simulate explicitly lepton events for each channel 
$i$ with fixed angular distribution in the individual $a+b$ system and then transform the lepton and photon 4-momenta to the $A+B$ system. For example, for the Dalitz decay of the $\eta$-meson, the lepton angular distribution in the rest frame of the $\eta$-meson has a form (2) with $B_{\eta}^{a b}=+1$ [10], where $\theta_{a b}$ is the angle between the lepton momentum $\vec{l}^{*}$ and the direction of $\vec{q}^{\eta}$, while $\vec{q}^{\eta}$ is the virtual photon momentum in the rest frame of the $\eta$. We also use that the distribution of the virtual photon momentum $\vec{q}^{\eta}$ in the rest frame of the $\eta$ is isotropic. Thus, for each $\eta$ event with energy $E_{\eta}$ and momentum $\vec{P}_{\eta}$ in the nucleus c.m.s. $A+B$ we generate lepton events distributed with respect to $\vec{q}^{\eta}$ according to Eq. (2) with $B_{\eta}^{a b}=+1$ in the lepton c.m.s. The next step then is the Lorentz transformation from the $\eta$ rest frame to the c.m.s. of $A+B: \vec{q}_{A B}=L\left(\frac{\vec{P}_{\eta}}{E_{\eta}}\right) \vec{q}_{\eta}$, where $q_{0_{\eta}}=\frac{m_{\eta}^{2}+M^{2}}{2 m_{\eta}},\left|\vec{q}_{\eta}\right|=\sqrt{\frac{\lambda\left(m_{\eta}^{2}, M^{2}, 0\right)}{2 m_{\eta}}}$. Finally we compute the angle $\theta_{A B}$ between $\vec{l}^{*}$ and $\vec{v}_{q}^{A B}$ as

$$
\cos \theta_{A B}=\frac{\overrightarrow{l^{*}} \cdot \vec{v}_{q}^{A B}}{\left|\overrightarrow{l^{*}}\right| \cdot\left|\vec{v}_{q}^{A B}\right|},
$$

where $\vec{v}_{q}^{A B}=\vec{q}^{A B} / q_{0}^{A B}$ and $\left|\overrightarrow{l^{*}}\right|=M / 2$ for fixed invariant mass $M$. Thus the angular distribution as a function of $\cos \theta_{A B}$ for all generated lepton events is recovered and $B_{\eta}$ can be computed via

$$
B_{\eta}^{A B}=\frac{W\left(\theta_{A B}=0^{\circ}\right)}{W\left(\theta_{A B}=90^{\circ}\right)}-1 .
$$

The weighted $<B_{\eta}>$ then follows from Eq. (6).

In a similar way we calculate the anisotropy coefficient for the pion annihilation channel by using that the anisotropy coefficient in the c.m.s. of the leptons (or pions) $10 B_{\pi^{+} \pi^{-}}^{a b}=$ -1 . For each pion annihilation event we then restore the virtual photon energy and momentum in the c.m.s. of $A+B: q_{0}^{A B}=E_{a}+E_{b}, \vec{q}^{A B}=\vec{p}_{a}+\vec{p}_{b}$, where $E_{a}, E_{b}, \vec{p}_{a}, \vec{p}_{b}$ are the energies and momenta of the pions in the $A+B$ c.m.s. Then we perform a Lorentz transformation for the pion momentum from the $A+B$ c.m.s. to the leptons c.m.s.: $\vec{p}_{a}^{*}=L\left(\frac{\vec{q}^{A B}}{q_{0}^{A B}}\right) \vec{p}_{a}$. In the lepton c.m.s. we generate the lepton events with $\left|\overrightarrow{l^{*}}\right|=M / 2$ $\left(M^{2}=q_{0}^{A B^{2}}-\vec{q}^{A B^{2}}\right)$ and angular distribution $W\left(\theta_{a b}\right)=1-\cos ^{2} \theta_{a b}$, where $\theta_{a b}$ is the angle between the $\vec{l}^{*}$ and the pion momentum $\vec{p}_{a}^{*}$ in the leptons c.m.s.; $\cos \theta_{a b}=\frac{\vec{l}^{*} \cdot \vec{p}_{a}^{*}}{\left|\overrightarrow{l^{*}} \cdot\right| \cdot \vec{p}_{a}^{*} \mid}$. For each selected lepton event the angle $\theta_{A B}$ can be calculated according to Eq. (11). The weighted coefficient $\left\langle B_{\pi^{+} \pi^{-}}>\right.$can be computed in the similar manner as in the $\eta$ case.

The anisotropy coefficient for the Dalitz decay of the $\omega$-meson, furthermore, is computed in analogy to the $\eta$ case, using the elementary coefficient $B_{\omega}^{a b}=+1$. 
We have tested our algorithm for BEVALAC energies and - within the statistical accuracy - reproduce the results from our previous paper [13], where an approximation was made to calculate the anisotropy coefficients for the Dalitz decays of $\Delta$ and $\eta$ : in 13 we have used the results of our microscopic calculations for the anisotropy coefficient that were already averaged over $\bar{q}^{a b}$ whereas, in principle, we need this degree of freedom to transform correctly the differential cross section from the $a+b$ to the $A+B$ c.m.s. Our discrete algorithm now allows to avoid this approximation; it is close to an experimental event by event analysis and includes the full hadron dynamics.

\section{Numerical results for $p+B e$ and $A+A$ reactions}

In this section we present the results of our calculations for the dilepton spectra and the anisotropy coefficient for the systems $p+B e, C a+C a, S+A u, A u+A u$ from BEVALAC to SPS energies without employing any medium effects for the mesons, i.e. $U_{m}^{S}=0, U_{m}^{\mu}=0$ in the set of transport equations (7) for all mesons.

In the Fig. 11 we show the computed weighted anisotropy coefficients $\left\langle B_{i}(M)\right\rangle$ for $p+B e$ and $\mathrm{Ca}+\mathrm{Ca}$ collisions at the bombarding energy of $2 \mathrm{GeV} / \mathrm{A}$ essentially confirming our previous results from [13] employing the averaged coefficients as described above. We note that the inclusive dilepton spectra for the reactions have been presented in Ref. [13] together with a "cocktail" decomposition. The main contributions arise from the $\eta$ and $\Delta$ Dalitz decays due to their large 'elementary' anisotropy coefficients and cross sections, respectively. The contribution from $p n$ bremsstrahlung is practically zero at all invariant masses due to a small 'elementary' anisotropy coefficient. The weighted coefficient from $\pi^{+} \pi^{-}$annihilation is rather small $(\approx 0.1)$ even for the $C a+C a$ reaction and decreases for $M \geq m_{\rho}$ due to the threshold behaviour of the cross section. However, compared to $<B_{\pi^{+} \pi^{-}}(M)>$ for $p+B e$, where pion annihilation is very low, a clear (but moderate) enhancement can be extracted.

Before going over to the presentation of the anisotropy coefficient at AGS energies $(\sim 10 \mathrm{GeV} / \mathrm{A})$ we show the differential multiplicity $d n_{e^{+} e^{-}} / d M$ for $p+B e$ and $A u+A u$ in order to demonstrate the relative contribution of the different channels employing an 'energy resolution' of $50 \mathrm{MeV}$ (Fig. 2). In the 'cocktail' plot the $\omega \rightarrow \pi e^{+} e^{-}$Dalitz decay,

$\eta$ Dalitz decay (' $\eta$ '), the pion annihilation channel $\left(' \pi^{+} \pi^{-}\right)$as well as the direct decay of the vector mesons (' $\rho, \omega, \Phi ')$ are displayed explicitly while the solid curves (denoted 
by 'all') represent the sum of all sources. The contributions from the $\Delta$ Dalitz decay and the bremsstrahlung channels are low by an order of magnitude compared to the $\eta$-channel and not plotted explicitly. Similar to the systems at BEVALAC energies the $\pi^{+} \pi^{-}$annihilation channel is of no importance for $p+B e$, but becomes the most significant sources for $M>0.4 \mathrm{GeV}$ in case of $A u+A u$ collisions. Furthermore, when scaling the spectra for $A u+A u$ by the number of projectile nucleons, we observe an additional enhancement in the $\Phi$-mass region which is due to the meson induced secondary collisions channels $K \bar{K} \rightarrow \Phi \rightarrow e^{+} e^{-}$and $\pi \rho \rightarrow \Phi \rightarrow e^{+} e^{-}$.

In Fig. 3 we show the weighted anisotropy coefficients $\left\langle B_{i}(M)\right\rangle$ for $p+B e$ and $A u+$ $A u$ collisions at $10 \mathrm{GeV} / \mathrm{A}$ using the same notations as in Fig. 2. The main contribution arises from the $\eta$-Dalitz decays whereas the contribution from the $\omega$-Dalitz decay is quite small due to its reduced cross section. The weighted anisotropy coefficient from pion annihilation for $p+B e$ is zero for the same reason. However, the $<B_{\pi^{+} \pi^{-}}(M)>$ for $A u+A u$ is zero because the angular distribution of the annihilating pions relative to the $\vec{q}^{A B}$ direction becomes practically isotropic at this energy which leads automatically to an isotropic dilepton spectrum. Obviously, this effect is related to our choice of the $z$-axis along the $\vec{q}^{A B}$. One can show, for example, that in the Gottfried-Jackson frame, the distribution of annihilating pions is anisotropic and $<B_{\pi^{+} \pi^{-}}(M)>$ is small and negative at this energy.

Before presenting our results for SPS energies we like to point out that the transport approach HSD provides a good reproduction of the pion rapidity and transverse energy distribution at SIS/BEVALAC and AGS energies [14 which ensures that the actual pion phase-space densities and thus the $\pi^{+} \pi^{-}$annihilation rate should be realistic. However, a definite proof for SPS is still lacking, since only rapidity distributions have been presented so far in comparison to the experimental data for $S+A u$ at $200 \mathrm{GeV} / \mathrm{A}$ in [15]. We thus show in Fig. $\mathbb{\theta}$ the calculated invariant cross section for $\pi^{0}$ mesons in the rapidity interval $2.1 \leq y \leq 2.9$ as a function of the transverse mass of the pions for $S+A u$ at $200 \mathrm{GeV} / \mathrm{A}$ in comparison with the experimental data of the WA80 collaboration [26]. As can be seen from Fig. Ð the results of our computation (open circles) are in a good agreement with the experimental data (full circles) also with respect to the transverse mass distribution.

The dilepton invariant mass spectra for $p+B e$ at $450 \mathrm{GeV} / \mathrm{A}$ and $S+A u$ at $200 \mathrm{GeV} / \mathrm{A}$ have been presented in [15]. For 'free' meson masses and formfactors the experimental cross section is slightly underestimated for invariant masses $0.3 \leq M \leq 0.45 \mathrm{GeV}$, which 
may be due to a dropping of the $\rho$-meson mass at finite baryon density [15, 27]. In the present work we do not explore this idea in more detail and calculate the dilepton spectra without any medium modifications.

In Fig. 5 we show the differential dilepton multiplicities for $A u+A u$ at $160 \mathrm{GeV} / \mathrm{A}$, a system that has recently been investigated experimentally at the SPS. Again the dominant contributions come from the $\eta$ Dalitz decay at small invariant masses and from the pion annihilation and direct decay of vector mesons at $M \sim m_{\rho}$.

In Fig. 6 we present the weighted anisotropy coefficients $<B_{i}(M)>$ for $p+B e$ collisions at $450 \mathrm{GeV} / \mathrm{A}, S+A u$ at $200 \mathrm{GeV} / \mathrm{A}$ and $A u+A u$ at $160 \mathrm{GeV} / \mathrm{A}$. For $p+B e$ the situation is similar to the previous cases at lower energies, the main contribution at small invariant masses comes from the $\eta$ Dalitz decay; $<B_{\pi^{+} \pi^{-}}(M)>$ is approximately zero due to the small cross section. For nucleus-nucleus collisions, however, the contribution of the pion annihilation channel becomes more essential. As seen from Fig. 6, contrary to the lower bombarding energies, the weighted anisotropy coefficient for pion annihilation is negative; an effect related to some differences in the pion dynamics at low and high energy as we will discuss below.

In order to explore this effect we use the result from [13] that the dilepton anisotropy coefficient $<B_{\pi^{+} \pi^{-}}(M)>$ is directly connected with the angular distribution of annihilating pions $W\left(M, \cos \theta_{\pi}\right)$ relative to $\vec{q}^{A B}$ :

$$
B_{\pi^{+} \pi^{-}}^{A B}(M)=\frac{\int d \cos \theta_{\pi} \tilde{A}\left(\theta_{\pi}\right) \tilde{B}\left(\theta_{\pi}\right) W\left(M, \cos \theta_{\pi}\right)}{\int d \cos \theta_{\pi} \tilde{A}\left(\theta_{\pi}\right) W\left(M, \cos \theta_{\pi}\right)} .
$$

The quantities $\tilde{A}\left(\theta_{\pi}\right), \tilde{B}\left(\theta_{\pi}\right)$ result from a Lorentz transformation from the angle $\theta_{a b}$ to $\theta_{A B}$ and read

$$
\tilde{A}\left(\theta_{\pi}\right)=1+\frac{B_{\pi^{+} \pi^{-}}^{a b}}{2} \sin ^{2} \theta_{\pi}, \quad \tilde{B}\left(\theta_{\pi}\right)=\frac{B_{\pi^{+} \pi^{-}}^{a b}}{2 \tilde{A}\left(\theta_{\pi}\right)}\left(3 \cos ^{2} \theta_{\pi}-1\right) .
$$

Here $B_{\pi^{+} \pi^{-}}^{a b}=-1$, while $\theta_{\pi}$ is the angle between the pion momentum $\vec{p}_{a}^{*}$ in the c.m.s. of $a+b$ and the direction of the vector $\vec{v}_{q}^{A B}$. In the $A+B$ c.m.s. $\theta_{\pi}$ can be written as

$$
\cos \theta_{\pi}=\frac{M \Delta E}{\sqrt{\left(M^{2}-4 m_{\pi}^{2}\right)\left(E^{2}-M^{2}\right)}}
$$

where $\Delta E=E_{a}-E_{b}, E=E_{a}+E_{b}$ are the relative and total energies of the annihilating pions with energy $E_{a}, E_{b}$ in the c.m.s. of $A+B$.

In Eq. (15) the $\pi^{+} \pi^{-}$angular distribution $W\left(M, \cos \theta_{\pi}\right)$ describes the energy and angular distribution of the pions that annihilate. Because of simple kinematical relations 
the distribution of the annihilating pions in $\Delta E$ and $E$ becomes broader with increasing bombarding energy. In line with Eq. (15) this leads to a transition from a distribution $W\left(M, \cos \theta_{\pi}\right)$ peaked at $90^{\circ}$ at low energies to a much flatter one at high energies or to a positive $B$ coefficient at small energies and a negative one at high energies.

To quantify our above arguments for the coefficient $<B_{\pi^{+} \pi^{-}}(M)>$ we show in Fig. 7 the distribution $W\left(M, \cos \theta_{\pi}\right)$, a contour plot of $\cos \theta_{\pi}$ in the plane $(\Delta E, E)$ at $M=$ $0.7 \mathrm{GeV}$ (see Eq. (15)), as well as contour plots of the density distribution $W(\Delta E, E)$ for $C a+C a$ at $2 \mathrm{GeV} / \mathrm{A}, A u+A u$ at $10 \mathrm{GeV} / \mathrm{A}$ and $S+A u$ at $200 \mathrm{GeV} / \mathrm{A}$. The value of $\cos \theta_{\pi}$ is zero at $\Delta E=0$ and goes to \pm 1 when increasing the absolute value of $\Delta E$ according to Eq. (15). First of all one can see a pronounced change in the shape of $W\left(M, \cos \theta_{\pi}\right)$ from $C a+C a$ at $2 \mathrm{GeV} / \mathrm{A}$ to isotropy for $A u+A u$ at $10 \mathrm{GeV} / \mathrm{A}$ and to a forward - backward peaked distribution for $S+A u$ at $200 \mathrm{GeV} / \mathrm{A}$. In the $(E, \Delta E)$-plane the maximum of $W(\Delta E, E)$ is located near $\Delta E=0$ at low energy which corresponds to $\cos \theta_{\pi}=0$. Thus, at low energy the probability for pions to annihilate at angle $\theta_{\pi}=90^{\circ}$

relative to the $\vec{q}^{A B}$ is larger than at $0^{\circ}, 180^{\circ}$; correspondingly $W\left(M, \cos \theta_{\pi}\right)$ has a maximum at $90^{\circ}$ (r.h.s). Furthermore, as seen from Fig. 目 the absolute value of the $W_{\max }(\Delta E, E)$ at $\Delta E=0$ decreases with increasing energy and the probability for annihilating pions with $\theta_{\pi}=0^{\circ}, 180^{\circ}$ increases. In other words, at high energies the annihilating pions have larger longitudinal than transverse momentum in the c.m.s. of leptons relative to $\vec{q}^{A B}$ (r.h.s.), and according to Eq. (13) the anisotropy coefficient $B_{\pi^{+} \pi^{-}}^{A B}$ becomes negative.

\section{Summary}

In this work we have presented a fully dynamical study of dilepton production in protonnucleus and nucleus-nucleus collisions from SIS/BEVALAC to SPS energies. Due to the parallel ensemble algorithm employed we have, furthermore, calculated dilepton angular anisotropies on an event by event basis for the channels $\eta \rightarrow \gamma e^{+} e^{-}, \omega \rightarrow \pi^{0} e^{+} e^{-}$, $\Delta \rightarrow N e^{+} e^{-}$as well as dilepton bremsstrahlung and $\pi^{+} \pi^{-}$annihilation. In all reactions we find the anisotropy coefficient $<B>$ to be dominated by the $\eta$-decay for invariant masses $M<0.4 \mathrm{GeV}$, while $<B>$ is practically zero for $M>0.5$ in $p+B e$ reactions at all bombarding energies. This situation changes significantly for nucleus-nucleus collisions showing a positive $\langle B\rangle$ from $\pi^{+} \pi^{-}$annihilation of about 0.1 at $2 \mathrm{GeV} / \mathrm{A}$, turning approximately zero at $10 \mathrm{GeV} / \mathrm{A}$ and becoming negative $<B>\approx-0.1$ at SPS energies. 
We could demonstrate that this energy variation is due to the different pion annihilation dynamics.

Thus, the calculated anisotropy coefficients for nucleus-nucleus collisions support our suggestion in Refs. 110, 11, 12 that the dilepton decay anisotropy may serve as an additional observable to decompose the dilepton spectra into the various sources and to obtain additional information on the reaction dynamics. We note, however, that our transport calculations correspond to the full acceptance for the lepton pairs. Especially cuts in the transverse momenta affect the anisotropies sensitively. This should be born in mind when analyzing experimental data with a limited acceptance.

\section{Acknowledgment}

The authors like to thank O.V. Teryaev and V.D. Toneev for the cooperation on earlier stages of this work, their critical remarks and valuable comments and discussions. Our special gratitude is to S.S. Shimanskij for stimulating suggestions and encouraging talks. We are grateful also to A.M. Baldin, A. Drees, A.A. Sibirtsev, S. Teis, A.I. Titov and Gy. Wolf for clarifying discussions. 


\section{References}

[1] G. Roche et al., Phys. Rev. Lett. 61 (1988) 1069

[2] C. Naudet et al., Phys. Rev. Lett. 62 (1989) 2652

[3] G. Roche et al., Phys. Lett. 226B (1989) 228

[4] G. Agakichiev et al., Phys. Rev. Lett. 75 (1995) 1272.

[5] T. Akesson et al., Z. Phys. C68 (1995) 47.

[6] M.A. Mazzoni et al., Nucl. Phys. A566 (1994) 95c.

[7] E.V. Shuryak, Phys. Rep. 61 (1980) 71.

[8] J. Badier et. al., Phys. Lett. B89 (1979) 145; K.J. Anderson et al., Phys. Rev. Lett. $42(1979) 944$.

[9] R. Strojnowski, Phys. Rep. 71 (1981) 1.

[10] E.L. Bratkovskaya, O.V. Teryaev and V.D. Toneev, Phys. Lett. B348 (1995) 283.

[11] E.L. Bratkovskaya, M. Schäfer, W. Cassing, U. Mosel, O.V. Teryaev, and V.D. Toneev, Phys. Lett. B348 (1995) 325.

[12] E. L. Bratkovskaya, W. Cassing, U. Mosel, O. V. Teryaev, A. I. Titov, and V. D. Toneev, Phys. Lett. B362 (1995) 17.

[13] E. L. Bratkovskaya, W. Cassing, U. Mosel, nucl-th/9601018, Phys. Lett. B (1996), in press.

[14] W. Ehehalt and W. Cassing, hep-ph/9507276, Nucl. Phys. A (1996), in press.

[15] W. Cassing, W. Ehehalt and C.M. Ko, Phys. Lett. B363 (1995) 35.

[16] W. Cassing, W. Ehehalt and I. Kralik, Phys. Lett. B (1996) in press.

[17] K. Gottfried and J.D. Jackson, Nuovo Cimento 33 (1964) 309.

[18] K.V. Vasavada, Phys. Rev. D16 (1977) 146.

[19] K. Weber et al., Nucl. Phys. A552 (1992) 713. 
[20] Gy. Wolf, G. Batko, W. Cassing, U. Mosel, K. Niita, and M. Schäfer, Nucl. Phys. A517 (1990) 615; Gy. Wolf, W. Cassing and U. Mosel, Nucl. Phys. A552 (1993) 549 ;

[21] B. Nilsson-Almqvist and E. Stenlund, Comp. Phys. Comm. 43 (1987) 387.

[22] Review of Particle Properties, Phys. Rev. D50 (1994) 1173.

[23] W. Cassing, V. Metag, U. Mosel, and K. Niita, Phys. Rep. 188 (1990) 363.

[24] L.G. Landsberg, Phys. Rep. 128 (1985) 301.

[25] V. Blobel et al., Phys. Lett. B48 (1974) 73.

[26] R. Albrecht et al., Phys. Lett. B361 (1995) 14.

[27] G.Q. Li, C.M. Ko and G.E. Brown, Phys. Rev. Lett. 75 (1995) 4007. 


\section{Figure captions}

Figure 1: The weighted anisotropy coefficients $\left\langle B_{i}(M)>\right.$ for $p+B e$ collisions at the bombarding energy of $2.1 \mathrm{GeV}$ and $\mathrm{Ca}+\mathrm{Ca}$ collisions at the bombarding energy of $2.0 \mathrm{GeV} / \mathrm{A}$. The " $\eta$ " denotes the contribution of the $\eta$-channel, the " $\Delta$ " labels the contribution of the $\Delta$ Dalitz decay, " $p n$ " the proton-neutron bremsstrahlung, and " $\pi^{+} \pi^{-}$" the pion annihilation channel. The solid curves (denoted by "all") show the sum of all sources.

Figure 2: The differential multiplicity $d n_{e^{+} e^{-}} / d M$ for $p+B e$ and central $A u+A u$ collisions at a bombarding energy of $10 \mathrm{GeV} / \mathrm{A}$. The " $\omega \rightarrow \pi e^{+} e^{- \text {" }}$ is the $\omega \rightarrow \pi e^{+} e^{-}$Dalitz decay, " $\rho$ ", " $\omega "$, " $\Phi "$ are the direct decays of the vector mesons. The other notations are the same as in Fig. 1.

Figure 3: The weighted anisotropy coefficients $\left\langle B_{i}(M)\right\rangle$ for $p+B e$ and central $A u+A u$ collisions at $10 \mathrm{GeV} / \mathrm{A}$. The notation is the same as in Fig. 2.

Figure 4: The calculated invariant cross section (open circles) for $\pi^{0}$ mesons in the rapidity interval $2.1 \leq y \leq 2.9$ as a function of the transverse pion mass for $S+A u$ at 200 $\mathrm{GeV} / \mathrm{A}$ in comparison with the experimental data (full circles) of the WA80 collaboration [26].

Figure 5: The differential multiplicity $d n_{e^{+} e^{-}} / d M$ for a central $A u+A u$ collision at 160 $\mathrm{GeV} / \mathrm{A}$. The notation is the same as in Fig. 2.

Figure 6: The weighted anisotropy coefficients $\left\langle B_{i}(M)>\right.$ for $p+B e$ collisions at 450 $\mathrm{GeV} / \mathrm{A}, S+A u$ at $200 \mathrm{GeV} / \mathrm{A}$ and $A u+A u$ at $160 \mathrm{GeV} / \mathrm{A}$. The notation is the same as in Fig. 2. 
Figure 7: (1.h.s.): The contour plot of $\cos \theta_{\pi}$ in the plane $(\Delta E, E)$ at $M=0.7 \mathrm{GeV}$ (see Eq. (15)), as well as the countor plots of the density distribution $W(\Delta E, E)$ for $C a+C a$ at $2 \mathrm{GeV} / \mathrm{A}, A u+A u$ at $10 \mathrm{GeV} / \mathrm{A}$ and $S+A u$ at $200 \mathrm{GeV} /$ A. (r.h.s.) The angular distribution $W(M, \cos \theta)$ for $0.65 \leq M \leq 0.75 \mathrm{GeV}$ for the same systems. The solid lines represent fits to the calculated distributions. 


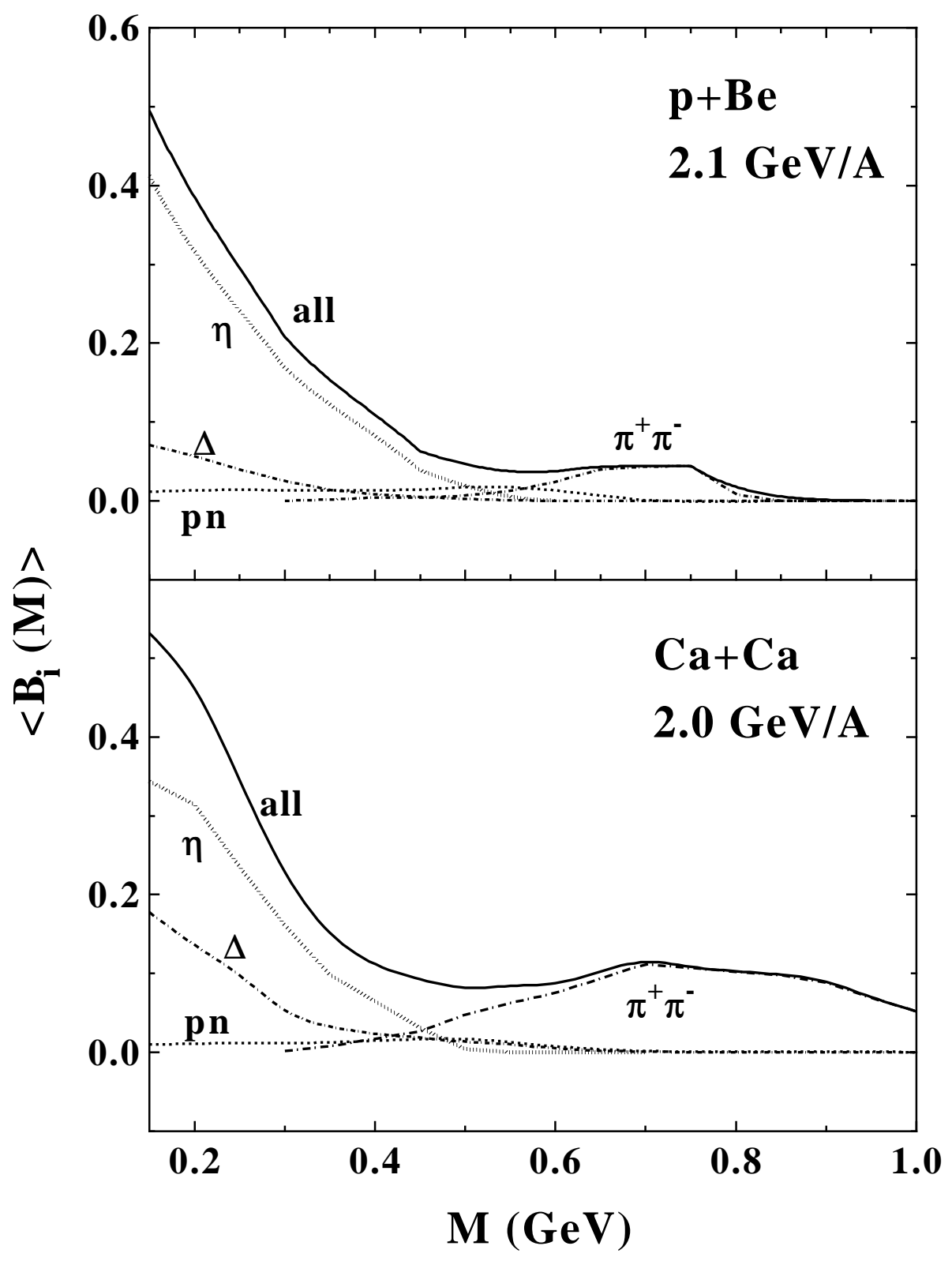

Fig. 1 

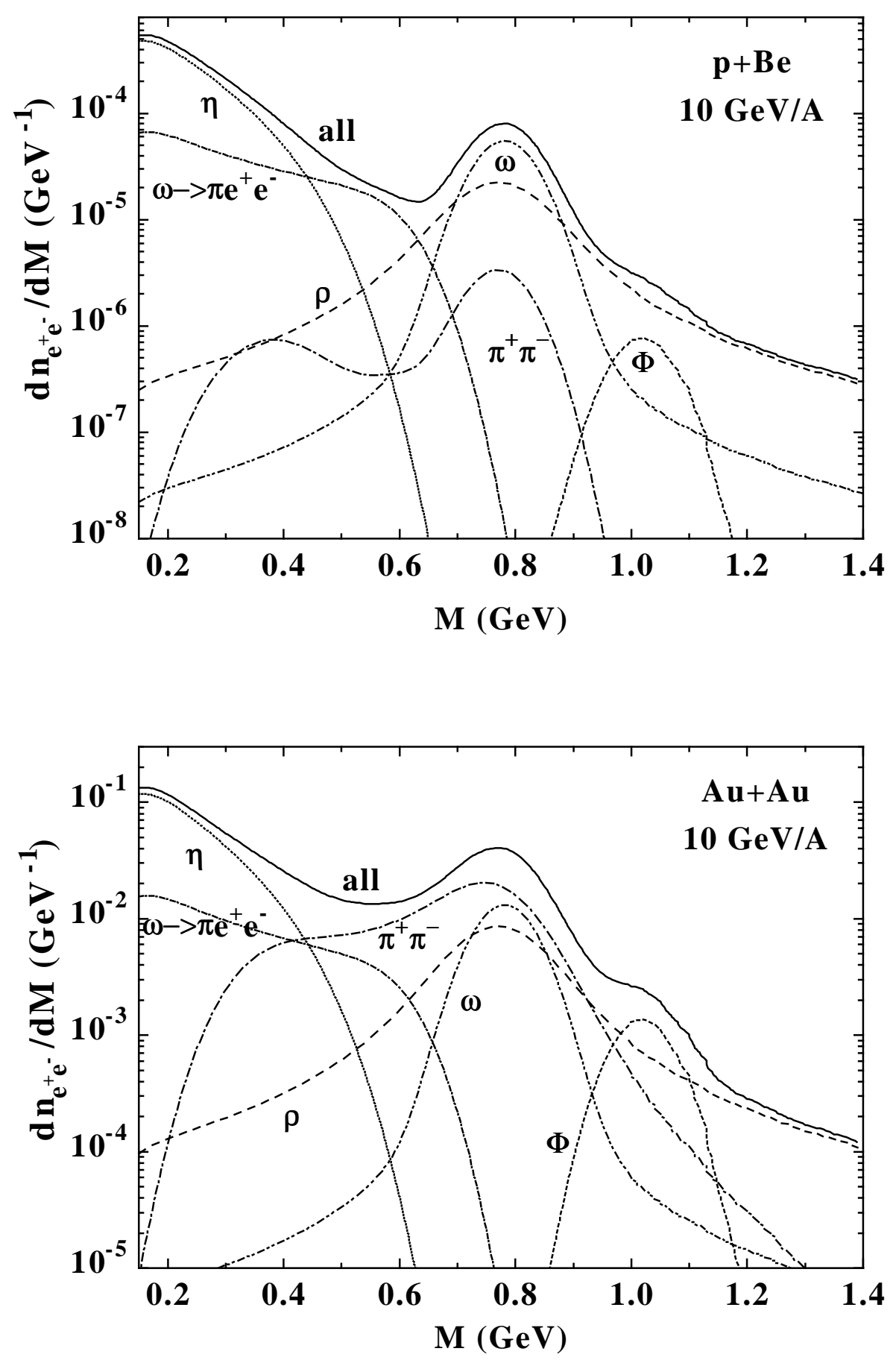

Fig. 2 


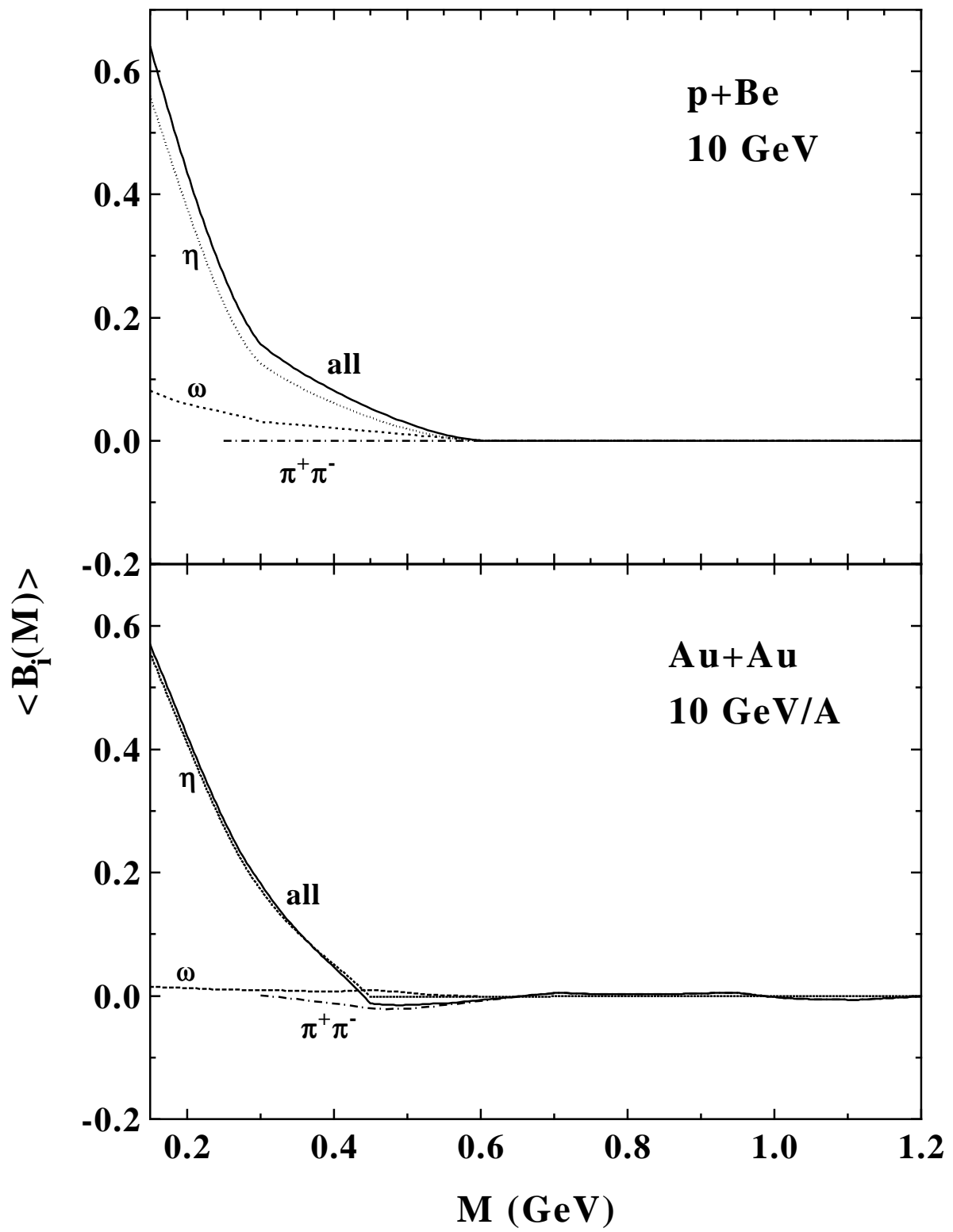

Fig. 3 


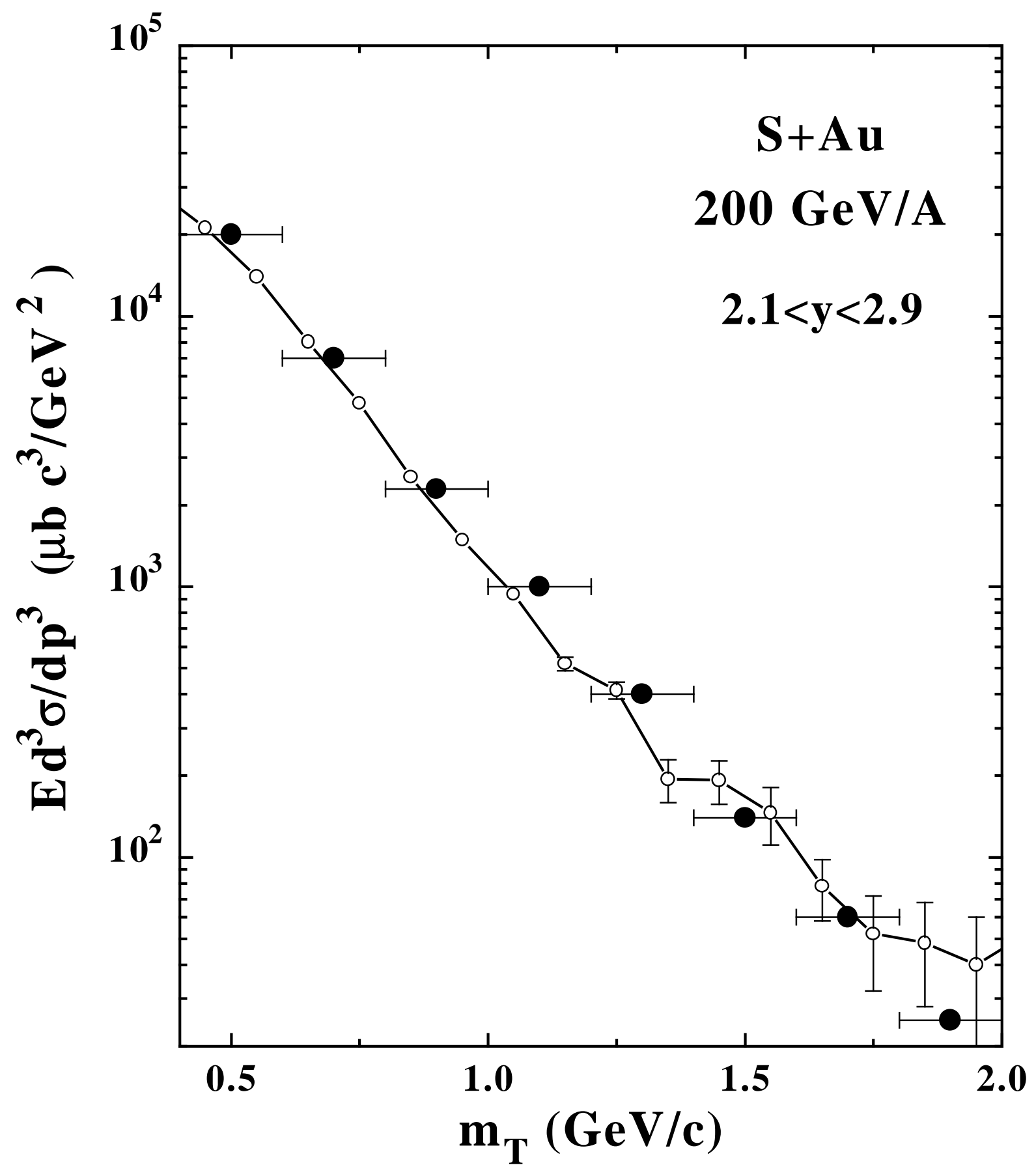

Fig. 4 


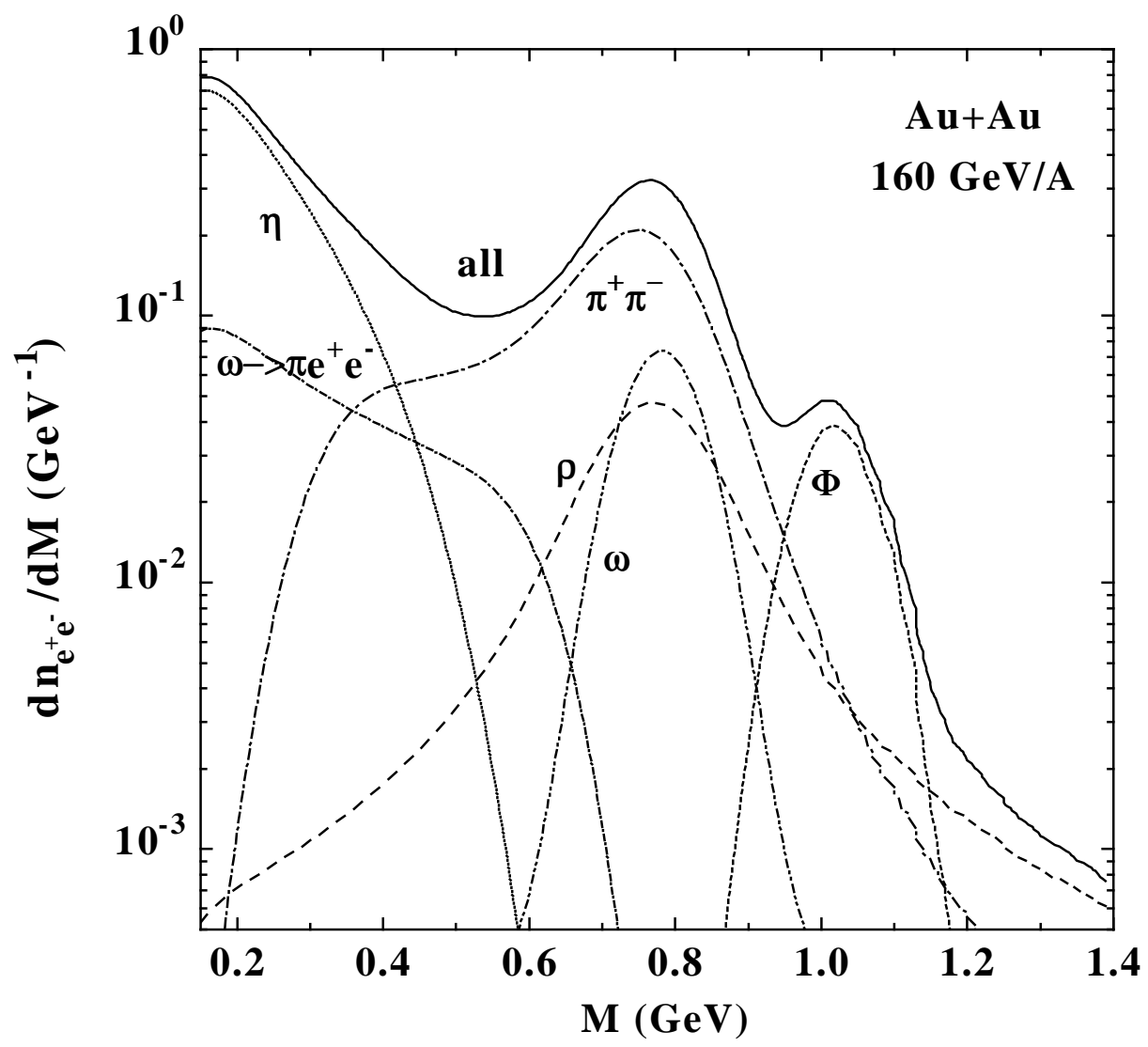

Fig. 5 


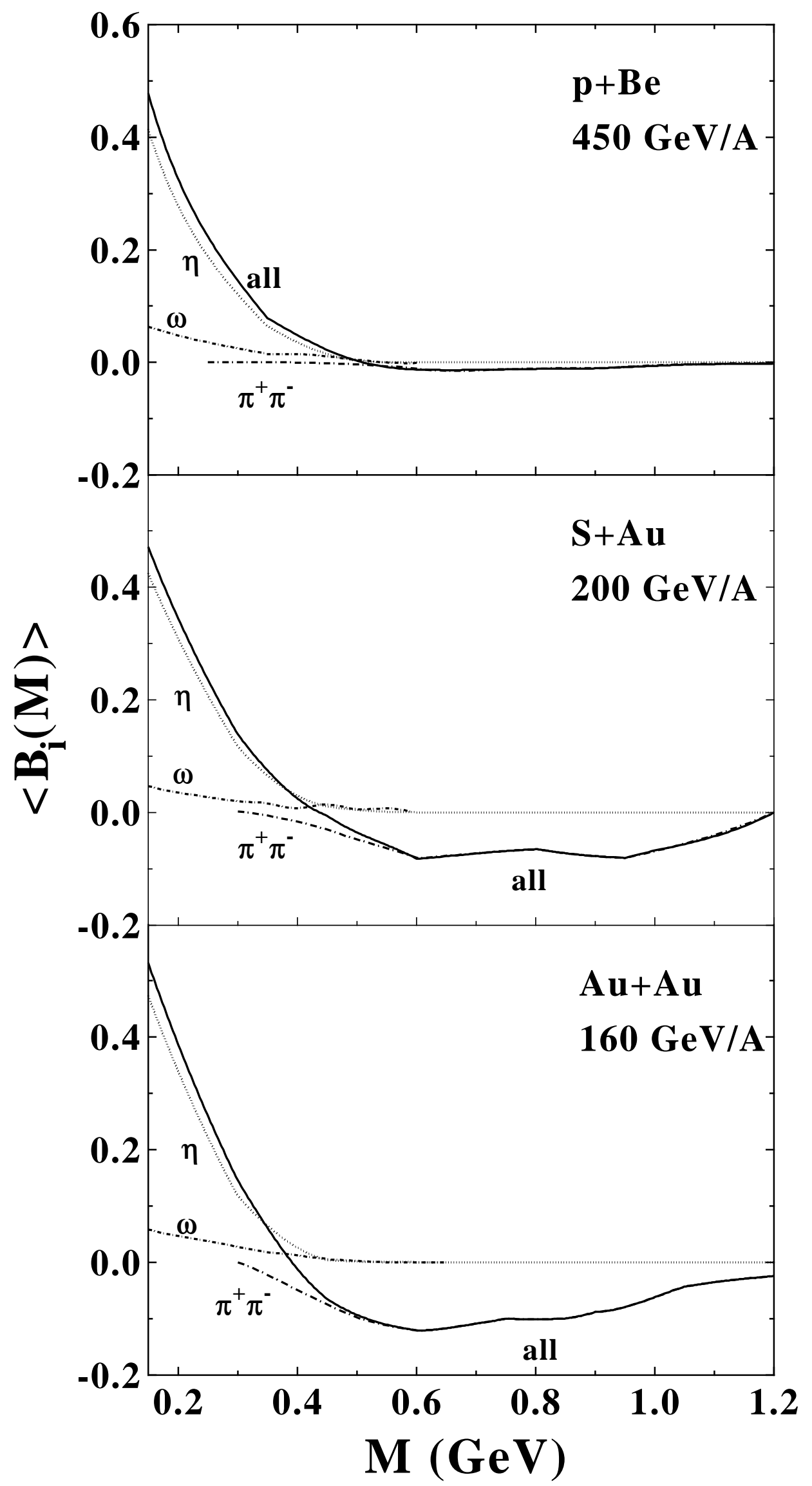

Fig. 6 

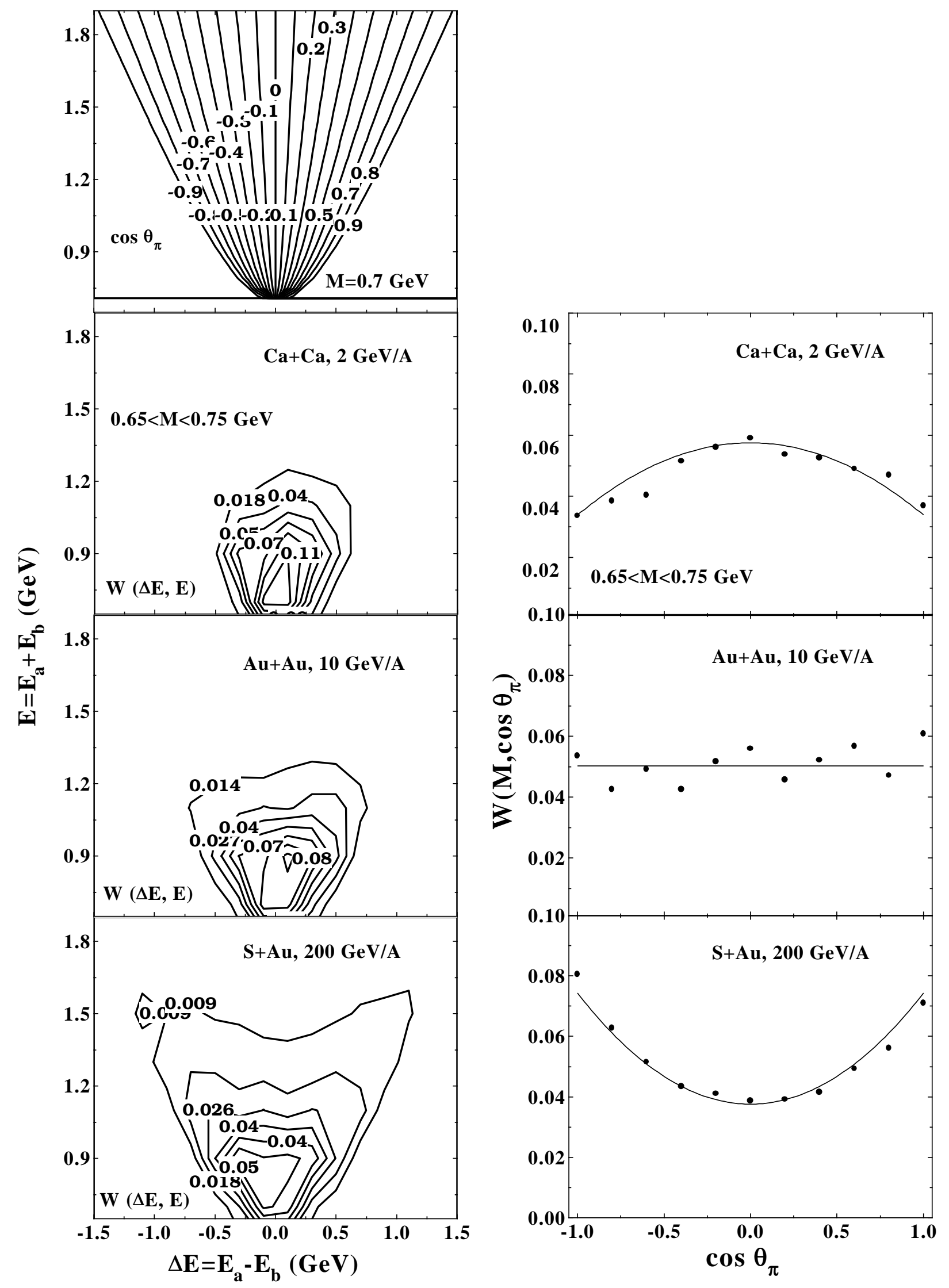

Fig. 7 